laparoscopic surgery $(P<0.05)$. Expression of endothelial cell-surface markers in tumors after laparotomy was approximately double that after laparoscopic surgery. There were no marked differences in circulating EPC levels or expression of endothelial cell-surface markers between mice that underwent laparoscopic surgery and controls.

The authors suggest that laparoscopic surgery might attenuate EPC mobilization by upregulating vascular endothelial growth factor and interleukin 6 to a lesser extent than laparotomy does. Laparoscopic tumor excision could reduce the incidence of metastases and benefit patients.

Original article Condon ET et al. (2007) Laparoscopic surgery protects against the oncologic adverse effects of open surgery by attenuating endothelial progenitor cell mobilization. Surg Endosc 21: 87-90

\section{Antibiotics are not always necessary for treatment of acute diverticulitis}

Antibiotic treatment for acute diverticulitis has several potential drawbacks; these include antibiotic resistance, toxicity, and changes to intestinal flora, as well as cost. To compare the outcomes of treatment with and without antibiotics, Hjern et al. conducted a retrospective analysis of 311 patients who were conservatively treated for acute diverticulitis between January 2000 and June 2002. The researchers found that antibiotics are not always necessary for the treatment of this condition.

Treatment included antibiotics for 118 patients (3 patients who did not respond also underwent surgery); for the remaining 193 patients, treatment consisted of careful observation, intravenous fluids and restriction of oral intake, to which antibiotics were added in the 7 cases of treatment failure. The decision to include or not to include antibiotics was taken by the attending surgeon. Patients treated with antibiotics were more likely to have fever at admission and higher levels of inflammatory markers than those who did not receive antibiotics; the mean hospital stay was also longer in the antibiotictreated group. Patient-reported time to recovery did not differ between the two groups, and recurrence rates, at 28-29\%, were almost identical. Regression analysis found that antibiotic treatment had no effect on risk of recurrence; the only factor associated with recurrence was a history of diverticular disease.

The results suggest that antibiotics can safely be omitted from treatment in patients with mild diverticulitis; the authors recommend prospective, randomized studies to compare antibiotic and non-antibiotic treatments for this condition.

Original article Hjern F et al. (2007) Conservative treatment of acute colonic diverticulitis: are antibiotics always mandatory? Scand J Gastroenterol 42: 41-47

\section{Colonoscopy failure rates are elevated in the afternoon}

Poor bowel preparation, a known predictor of incomplete or difficult colonoscopy, has been linked to colonoscopies scheduled late in the day. Physician fatigue has also been suggested to affect completion rates.

To determine the influence of scheduling on completion rates and adequacy of bowel preparation, Sanaka et al. retrospectively reviewed reports from 2,083 consecutive outpatient colonoscopies (1,084 morning, 999 afternoon). All patients' bowel preparation comprised $1 \mathrm{gal}-$ Ion (3.79I) of polyethylene-glycol-electrolyte solution, taken over $4 \mathrm{~h}$ during the evening before the day of colonoscopy.

Inadequate bowel preparation was more common for afternoon than morning colonoscopies ( $19.7 \%$ versus $15.4 \%, P=0.01)$. The incompletion rate was significantly higher in the afternoon than the morning $(6.5 \%$ versus $4.1 \%, P=0.01)$, even when colonoscopies terminated because of poor bowel preparation were excluded $(5 \%$ versus $3.2 \%, P=0.04$ ).

Scheduling colonoscopies in the afternoon might, therefore, be an independent predictor of incomplete colonoscopy and inadequate bowel preparation. The role of physician fatigue remains unclear-although morning completion rates were higher than afternoon completion rates or six of the eight endoscopists who took part in the study, the difference only reached statistical significance for one endoscopist. The authors suggest that scheduling colonoscopies in the morning could improve patient satisfaction and result in health-care cost savings (e.g. by avoiding repeat or earlier than recommended next colonoscopy). They acknowledge that alternative bowel preparation regimens could improve bowel preparation quality for afternoon 